\title{
Smart grid in the context of industry 4.0: an overview of communications technologies and challenges
}

\author{
Noor Ahmed Qarabsh ${ }^{1}$, Sana Sabah Sabry ${ }^{2}$, Haneen Ahmed Qarabash ${ }^{3}$ \\ ${ }^{1,2}$ College of Engineering, University Of Information Technology and Communications \\ ${ }^{3}$ College of science, University of Baghdad
}

\begin{tabular}{l} 
Article Info \\
\hline Article history: \\
Received Jul 26, 2019 \\
Revised Oct 25, 2019 \\
Accepted Nov 27, 2019 \\
\hline
\end{tabular}

Keywords:

Big data

Cyber-physical systems

Industry 4.0

Internet of things

Smart grid

\begin{abstract}
The recent advances in technology, the increased dependence on electrical energy and the emergence of the fourth industrial revolution (Industry 4.0) were all factors in the increased need for smart, efficient and reliable energy systems. This introduced the concept of the Smart Grid (SG). A SG is a potential replacement for older power grids, capable of adapting and distributing energy based on demand. SG systems are complex. They combine various components and have high requirements for real time reliable operation. This paper attempts to provide an overview of SG systems, by outlining SG architecture and various components. It also introduces communication technologies, integration and network management tools that are involved in SG systems. In addition, the paper highlights challenges and issues that need to be addressed for a successful implementation of SG. Finally, we provide suggestions for future research directions.
\end{abstract}

Copyright $(2020$ Institute of Advanced Engineering and Science. All rights reserved.

\section{Corresponding Author:}

Noor Ahmed Qarabash,

Collge of Engineering,

University Of Information Technology And Communications,

Baghdad, Iraq.

Email: noor.ahmed@uoitc.edu.iq

\section{INTRODUCTION}

The fourth industrial revolution, known as Industry 4.0, has received massive attention and extensive discussion from researchers and manufacturers. It is based on the use of smart production processes and close to full automation. It aims to enhance the manufacturing process, enable rapid growth of industry and provide supply and demand integration. The Internet of Things (IoT), big data analytics, Cyber Physical Systems (CPS) and automation are the major components of industry 4.0. People and resources are now moving away from a central approach toward the process of decentralized production [1].

With the constant increase of electricity prices, changes of earth's climate and the exhaustion of energy resources, the traditional power grid does not have the capacity to counter the increased demand for power to support advanced technology and industrial innovation [2]. The smart grid (SG) emerged as a suitable solution for addressing these challenges; Smart Grids enable the fourth stage of the industrial revolution, known as Smart Grid Industry 4.0 (SGI 4.0). In SGI 4.0, Information and Communications Technologies (ICT) can play a major role in increasing reliability, stability and efficiency compared to the traditional grid [3].

The concept of industry 4.0 is not limited to the factory but also encompasses the entire life cycle of the product, from production, supplier to the end user. The automation of this life cycle can be achieved by the utilization of ICTs such as IoT, cloud computing, machine learning and CPS. Smart grids play a role in every step of this product life cycle. Figure 1 shows the specific function of the smart grid to supply energy for the smart factory throughout the life cycle of the product between elements of different systems. SGI 4.0 
keeps track of all the relevant information about the flow of electricity in the plant. Data provided by smart meters and consumption statistics, can be used via machine learning to provide advanced decision making.

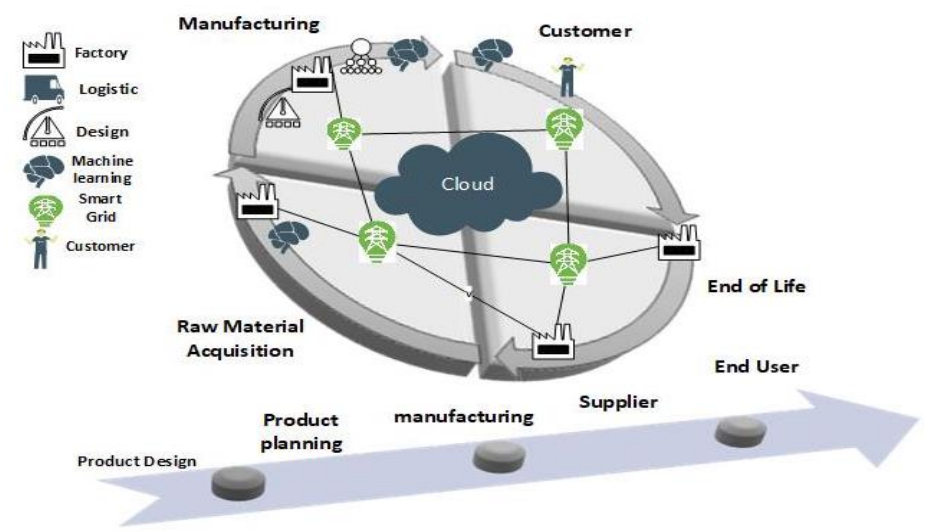

Figure 1. Product life cycle [4]

There are currently several Smart grid projects, in the European Union [5], China, US, and many other countries all at varying levels of integration and ranging in scale. It is expected that more countries will move in that direction since SGs can decrease carbon dioxide (CO2) and other greenhouse gas emissions. The global movement away from fossil-fuel toward renewable environmentally friendly, energy resources (green energy) such as wind, vibration, solar, etc. is enabled by SG systems [6]. It is also capable of reducing waste in energy production while increasing reliability by providing energy level that closely match demand instead of producing more than required causing unnecessary waste, or less than needed it causing shortages [7]. With the utilization machine learning, SG are enable to make important decisions based on the demand for energy, such as pricing in real-time, automated maintenance, scheduling of power usage and optimization of energy consumption. This allows Smart Grids to improve efficiency and enable power systems to operate independently with less human interference. Thus, in a Smart grid system there is a realtime interconnection between worker, customer and supplier with information exchange, building a high flexible power generation model [8].

The relationship between Industry 4.0 and SG can be symbyotic, where the smart grid can support effiecint energy systems that enable the industry. The industry can in turn provide more compatible enviroment for smart energy consumption. The shift from traditional Power Grid (PG) to the next generation of SGI 4.0 systems is a difficult task and requires robust designing and compatible infrastructure of communications network, to overcome any drawbacks related to the existing PG [9]. Furthermore, SGs are usually a combination of several systems and technologies, making it an intersting area of research and development. For researchers interested in this topic, it is necessary to have a general understanding of all these different aspects. Thus in this paper we will discuss the main concepts regarding SG, its architecture, enabling ICTs and current communications technologies used in SG from the perspective of industry 4.0.

There are some surveys and studies about SG [1, 6, 9-11] that cover a wide range of topics such as communications networks, architecture, security issues, or impact. However most of them are focused on one or two aspects, and they don't cover the context of Industry 4.0. Therefore, the purpose of this study is to provide researchers with a broad basic understanding of SG and offer resoucers to further explore the topic of SG, by discussing SGI 4.0, its architecture, advances in ICTs that enable it and challenges involved with its implementation, as well as future research direction in the field of SGI 4.0.

The rest of this paper is structured as follows: Section II presents a detailed overview of SGI 4.0 paradigm architecture while section III discusses the components of smart grids, focusing on IoT, CPS and cloud computing. Section IV presents an overview of communications technologies in SGI 4.0 while challenges with SGI4.0 were discussed in section V. Finally, sections VI and VII provide future research directions and conclusions.

\section{SMART GRID ARCHITECTURE}

Designing a robust communications network infrastructure is important for SGI4.0 to achieve reliable and efficient operation. There are multiple SG architecture models but they all follow a similar multilayered structure [12] Defines the three main layers as: 
a. Power systems layer: responsible for generating and delivering electrical energy to the users, similar to a traditional PG.

b. Communications layer: which provides interconnection between all the system components by collecting data from sensors and end user interfaces to transmit them to data centers and vice versa.

c. Applications layer: in this layer Information is processed to issue monitoring and control messages, as well as using the data for applications such as demand management, automatic meter reading and detection of fraud or misuse. The three layers are illustrated in Figure 2.

SG systems depend on the use of a number of networks that vary in size and location [13-14], such as:

a. Home Area Network (HAN) or customer area network, which connects smart appliances and devices to a smart meter inside the house. They have a short range and capable of reliably communicating with low data rates. Thus, they are to lower implementation costs and energy consumption.

b. Building Area Network, (BAN): similar to a HAN but covers larger buildings and can consist of multiple smaller networks.

c. Industry Area Network (IAN), like BANs but more complex and specialized for factories and industrial buildings.

d. Neighborhood area Network (NAN) responsible for connecting HANs, BANs, and IANs to WAN and metering data aggregation from thousands of smart meters. Due to the larger scope, NANs require higher data rates.

e. Wide Area Network (WAN), it is utilized by NANs to forward the electricity reports to the main control center. WANs require very high data rate and long coverage distance. Optical networks are commonly used as communications medium.

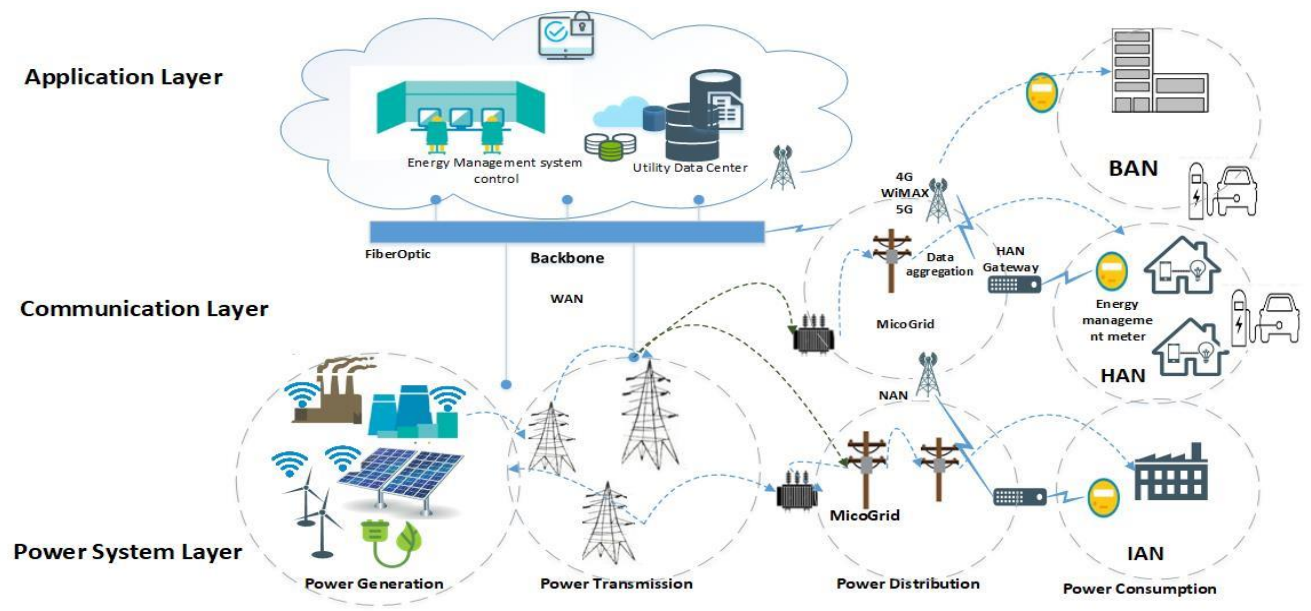

Figure 2. Smart Grid Architecture

\section{SMART GRID COMPONENTS}

Smart Grids are complex systems, consisting of various components and ICTs. Below is a discussion of some of the major technologies that constitute a component in any SG system.

\subsection{Internet of Things}

A network that connects any object to the internet via an exchange protocols to communicate monitoring, management, tracking and identification information between different smart devices is known as the Internet of things IoT). It has become the focus of research in various applications over the last couple of years, and has allowed for connecting a multitude of network embedded devices used in daily life to the internet. IoT is also considered revolutionary in that it adds functionality to existing network systems and allow them to provide solutions to time critical applications in many fields such as healthcare, manufacturing, logistics, military retail etc. [15].

IoT technology plays a key role in SGs, as it enables the transfer of data between the various components of SG efficiently. Blackout prevention and power reduction are IoT systems' primary applications in a smart grid. IoT, and advanced sensing technologies are required for lowering the impact of natural disasters, improving reliability of energy transmission lines and reducing further loss of power [16]. 
For the SG to achieve successful connectivity between users and applications some components are required, such as: sensors, smart energy meters, smart inverters for applications using solar energy, grid monitoring controls, substation feeders and network interfaces, etc. All of these components collectively work to transfer data accurately in real time [10].

\subsection{Cyber Physical Systems}

A Cyber-Physical System (CPS) is a system that integrates cyber and physical elements effectively. An ideal CPS consists of a computing system, networking tools, and physical components such as sensors. The physical aspects of a smart grid is monitored by controllers ; by connecting the sensors via a communications network, it is possible to keep track of the overall status of the smart grid and its working conditions, the sensors send all relevant data to the controllers to take action [17].

Smart grids integrate the electricity network infrastructure (physical systems) and the cyber systems (sensors, actuators,...etc.), and show CPS characteristics such as virtual and real world integration in a dynamic environment where different scenarios from the power grid (physical system) are fed to CPS for simulation mode adjustment to influence how the physical system performs in future times [18]. CPS technologies allow the smart grid to perform real-time analysis and measurement to improve decision making capability and improve power consumption, safety and cost reduction.

\subsection{Cloud Computing}

Cloud computing provides applications and services with data storage and processing capability over the internet, Cloud omputing pools resources to eliminate the need for dedicated physical systems and can allow for higher levels of automation. According to [19] this can offer 3 main benefits: First, Infrastructure cost reduction since the resources are already in place. Second Outsoursinng maintenance, which lowers both cost and risk. Third Scalability and ease of implementation, since upgrades can be applied without disruption. Cloud computing is built around offering 'things' as services, which gives us the three main modules of Cloud services [20]:

a. Infrastructure as Service (IaaS): In this model virtual infrastructure is provided for a range of functiounality, most notiably data storage, to contain the huge amount of user data and virtual machines that serve as data centers for the grid.

b. Platform as a Service (Paas) : This model offers developers resources to develop applications and run them on virtual platforms. Since the cloud has unlimited resouces this can simplfy software developmet and expand the range of applications that can be developed for the grid.

c. Software as a Service (SaaS) : This model gives users an interface to built in cloud applications. This could open the door to developing smart grid applications that involve user input and prefernces in energy distribution by giving them an interface to interact with the grid, without any configuration or instalation.

Cloud computing has been instrumental in allowing the smart grid to achieve real time data storage and processing it also reduces the costs required to the grid to expand without compromising availability, and it will continue to play a role in its development moving forward.

\section{COMMUNICATION TECHNOLOGIES In SGI4.0}

Data transmission and communication is an essensial part of the SG operation, thus it utilizes a range of communications technologies; generally, these technologies are not dedicated for the grid, they were developed for various ICT application and were co-opted for the smart grid due to their effectiveness. The grid must be able send and receive information from smart meters, and transfer them for long distances to be processed. This can be done using existing transmission technologies. The Smart grid also needs to have proper communication with legacy power grids using integration technologies, lastly, there are network protocols and architectures that can be used to support the smart grid operations. The following is a brief explanation of the most recent communication technologies commonly used in the smart grid.

\subsection{Data Transmission Technology}

Data transmission technologies are essential in a smart grid to deliver user data and instruction message between the various parts of the grid. These can be wired such as Fiber, or wireless, such as LPWAN, Zigbee, etc. Wireless technologies are usually preferred due to their simplicity and ease of implementation, but they are vulnerable to interference and require constant power charging or battery replacement [17].

a. Fiber: Fiber optics is a high-speed, high- capacity data transmission medium, it is quite costly to implement, so it is only used in situations where a high data rate for long distances is essential, that is why it's most commonly used in backbone networks [18]. 
b. Cellular Network: commonly used for smart phones, cellular technology use microwaves and triangulation to transmit data, the 3rd and 4th generation, known as $3 \mathrm{G}$ and $4 \mathrm{G}$ respectively are still widely used for mobile communication, however: $5 \mathrm{G}$, or the fifth generation of cellular technology is more promising for current and future applications in the smart grid it was developed to deliver high data rates combined with low message return time (latency) as law as one microsecond, and to support for time critical systems [21], which makes it ideal for SG. 5G is also a still evolving technology with high potential for future advancement in the next few years, it is expected to become more adaptable for smart grid metering applications and other WAN and NAN applications [17].

c. Zigbee: A wireless technology that commonly used in wireless sensor networks (WSN) and has been used in smart meter applications, Zigbee devices use a relay system to transfer message from one device to the other, it supports multiple network topologies and is a low-energy, low-cost solution, with the disadvantage of having a low bit rate, which is acceptable when it comes to smart meters communicating with HANs, since these are low range applications [22] .

d. Low Power Wide Area Network (LPWAN) The smart grid requires the transfer of data on a large scale particularly between NANs and WAN, LPWAN protocols have the ability to transfer data in an energy efficient way, at low data rates, and for long distances, the two most commonly used LPWAN protocols are LoRaWAN and Sigfox [23]. LoRaWAN is a Highly scalable wide area network protocol, that allows two way communication between smart devices, its range covers $2-5 \mathrm{Km}$ in urban areas and up to $15 \mathrm{Km}$ in suburban areas, with a data rate between 0.3 and $50 \mathrm{Kbps}$ [24]. This protocol was specifically developed to allow multiple IoT devices and applications to communicate over long distances, so it offers multi-tenancy and multiple network domains [25]. Sigfox is a protocol that operates in a similar way to cellular networks. It utilizes long waves to achieve a wide range up to $1000 \mathrm{KM}$. It has a small bandwidth of 12 bytes per message, 140 messages per day, which can be a disadvantage, though it is an acceptable range for home devices like smart meters that only need to send periodical messages. In addition the low bandwidth offers the advantage of lower noise effect so the system can operate at low power, about $0.1 \%$ of that of modern cell phone devices [25].

\subsection{Integration Technology}

There are several protocols developed for legacy power systems that offer adaptability to employing a smart grid and allow it to integrate with existing power grids, such as the DNP3(Distributed Network Protocol) and IEC 61850 which are a legacy system communication protocols that support integration, DNP3 is used for communication between a command center and a substation. IEC 61850 on the other hand is used for communication within a substation itself, hence those two protocols are often integrated together using mapping methods [26].

A more unified architecture was proposed by the OPC foundation, known as Open Platform Communications Unified Architecture (OPC-UA) this architecture offers a framework that provides an interface for enabling different devices, to communicate, sending and receiving command and control messages in a clientlserver model that is fully automated [27] The application of this architecture in a smart grid context was researched by [28], which found it to be suitable for increasing interoperability, i.e. OPC UA's main function, furthermore it is capable of increasing security at a higher level, since this architecture supports multiple security and encryption standards.

\subsection{Network Management Technologies}

The smart grid requirements of high speed and reliability has dictated the need for management protocols and technologies that manage configuration in a way that sustain efficiency throughout the grid. An example of this is SDN, (Software defined Network) A recent networking paradigm for centralized network control through an SDN controller device, that allows the local networking devices to take forwarding and routing decisions faster and in a more flexible manner, without the need for manual configuration. Employing SDNs in Smart grid systems can make them more efficient in data management and better able to react to failures or attacks, thus making it more capable of maintaining its critical functionality at all times [29].

Another two concepts that can improve smart grid management are Softwarization and virtualization, Softwarization is a paradigm where functionality is employed on a software level rather than hardware, this makes it easily reconfigurable, and more flexible to new additions and interacting with different technologies, Virtualization further increases flexibility by creating virtual network components (hardware, systems and resources) eliminating the need for changing the hardware components [30] both concepts are achievable using cloud services. 


\section{SGI 4.0 CHALLENGES}

Smart grid systems are complex, sophisticated, and they combine many interconnected components. These various components and the emerging nature of smart grids has introduced several challenges that must be addressed to ensure a robust, reliable and scalable grid. This section briefly presents the main challenges commonly discussed in literature.

\subsection{Communication System Challenges}

Research by [31] Summarizes communication system challenges in 3 main issues, interference, the need for common standards and data transmission rates.

a. Interference can be caused by home devices' signals that interfere with smart meters, or by harmonics emission in the grid itself. Interference can be addressed using interference detection and channel switching techniques. [32]

b. Standards for the smart grid are necessary to provide a framework for all the different components of the grid to work together. There are current efforts by various organizations, such as IEEE, or the American National Standards Institute, to develop such standards.

c. Data transmission rates can be managed by choosing the correct transmission protocol for each application.

\subsection{Big Data Challenges}

Big data refers to the huge amounts of data produced by modern information systems and the processing power required to analyze and store that data. It is a known concept in ICTs that introduces a number of challenges that need to be addressed. Following is a brief discussion of some of the challenges that smart grid systems face in regard to big data.

a. Real-time applications: Smart Grids are meant to adapt to the consumers consumption levels, which is constantly changing, this requires real-time data collection from a large number of smart meters at varying rates. The grid must also be able to process that data and execute changes based on it in a near real-time operation. [33] Suggests that some of the methods developed for IoT applications to address this issue can also be applied to the smart grid. For example, using predictive algorithms on house appliances to determine the levels of data to be expected from a smart meter at any time of the day which would help allocate processing resources accordingly.

b. Heterogeneous Data: The grid receives data from a number of different sources and in different formats, for example usage data, monitoring data, capacity levels, error message, authentication messages, metadata, etc. This data originates from different sources such as meters, sensors, actuators, stations, smart home devices, historical data, mobile applications and others. This is known as heterogeneity of data, meaning the grid has to handle structured, semi-structured, and unstructured data at the same time. There are several techniques to address this problem, such as data integration, data fusion and development of standardized software solutions that unify data formats across different devices [34].

c. Data compression and visualization: The data collected in the grid require storage and could also provide valuable analytic information, requiring extra processing. Compression methods should be efficient and work in real-time. Visualization can present the data in understandable graphs and charts. The choice of the right visualization method and presenting the data in the right way is a difficult process that needs to be considered carefully. Both compression and visualization require more research and development of standardized methods [34].

\subsection{Challenges in Cloud Computing}

Cloud computing technology offers an efficient solution for processing data in the smart grid, but there are some reliability issues when working in this context that should be accounted for. First, there are security concerns due to the lack of consistent policies regarding data location which can compromise privacy. Second, the lack of disaster recovery plans, which can compromise availability. Other concerns include international laws in regards to data stored at different countries, error management, and data rates. Other issues related to CC are Cost, compatibility and quality of service [35].

Typically, those challenges must be addressed through careful selection of cloud service providers that offer support as well as drafting agreements with said providers to ensure that international laws are upheld and service quality as maintained.

\subsection{Security Challenges}

Since smart grid systems are cyber-physical, they are vulnerable to cyber-attacks that can affect physical systems, making any security threat dangerous and highly impactful. Furthermore, there are many economic and political reasons that motivate targeting the smart grid in order to compromise the vital energy 
market. There is a wide range of threats to the security of the grid. [36] notes that different sources classify threats and attacks based on different variables., for example the exploited device (Sensors, Network devices, smart meters, etc.), the system architecture layers they target (communication layer, application layer, etc.), or on the security objective they disrupt (integrity, availability, confidentiality). Some attacks identified by researchers can describe multiple variations, for example Denial of service (DoS) attack can be an umbrella term for Jamming or flooding, at any level of the grid. Figure 3 summarizes the challenges in SGI 4.0 systems and applications.

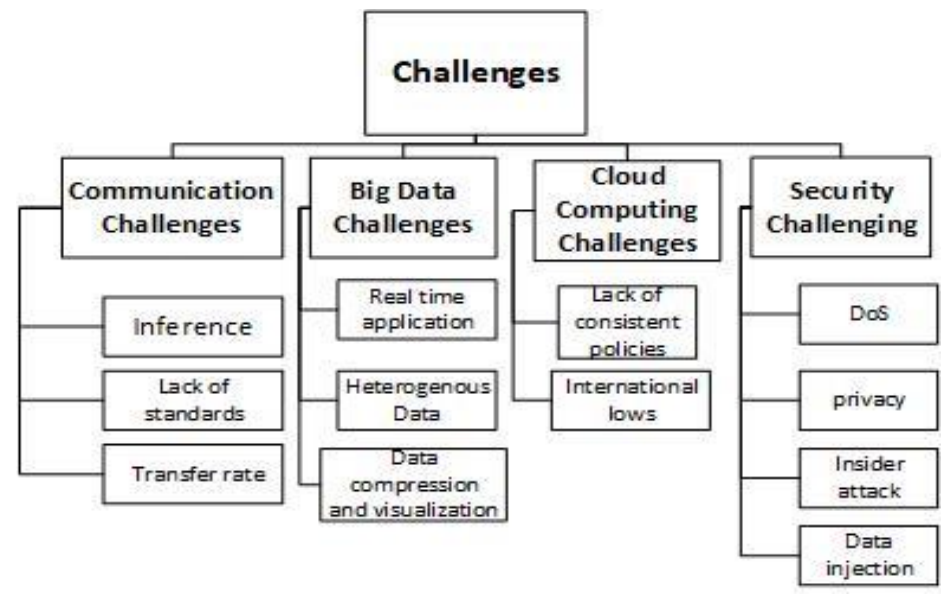

Figure 3. SGI 4.0 challenges

The following is a brief discussion of the main threats to the grid.

a. DoS attacks: Denial of service refers to a large number of attacks that cause a system to become overwhelmed and render it incapable of providing services required. This can be particularly threatening to a smart grid system because of its real-time operations. The control messages in the system are time critical and a few seconds delay can compromise all system operations. Advanced DoS attacks are hard to detect because they are disguised as legitimate traffic or are taking over trusted sources that it becomes hard to block all attacks. Furthermore, such attacks can come from different sources so it is impossible for one or several service providers to control. To reduce the impact of such attacks, the development of cyber resilient systems capable of withstanding multiple relentless attacks is important [37]. Another mothod that can be used to reduce DDos attacks is IP fast hopping, which disguses the true IP address of a service so that it becomes harder to target by attackers [38].

b. Data injection: These attacks occur when a malicious entity alters codes and database entries to disrupt the system. The smart grid is vulnerable to this kind of attack because of the various devices connected to it that provide access points for attackers. Moreover, this kind of attack can have a wide range of effects on power systems, from disrupting the real-time nature of the grid, to committing fraud by manipulating smart meter readings and power pricing [39]. Data injection attacks are hard to detect by nature and it's is better to prevent them, using smart security systems with dedicated authentication to limit the probability of these attacks [40].

c. Privacy: Smart Grid systems collect a range of user data, such as location, payment information power usage and preferences. This information can be used maliciously to track and harm users. Even information that might seem irrelevant can be used against the customer. For example, a user's power usage trends can help predict what time they leave the house thus allowing thieves to target their house, or an electrical devices company can use preferences of the user to advertise directly to them and gain advantage. That is why protecting users' privacy and information is very important. Multiple methods can be used to preserve privacy in smart grids, such as anonymization of data, masking sources, encryption and aggregating data using various methods that disassociate users from data [41].

d. Insider attacks: Insider threats, or attacks committed by anyone with legal access to the system, are dangerous because traditional security measures such as firewalls and passwords cannot stop them from causing damage. Some hiring practices like background check can help limit insider threats. There are also some technological solutions such as anomaly detection systems that can spot any irregular behavior, or the use of authenticated access control via gateway devices and software solutions [42]. 


\section{FUTURE RESEARCH DIRECTIONS}

The smart grid is still rapidly evolving and adapting to migration from legacy power grids. Communication and data processing technologies are also constantly advancing and progressing. It is hard to predict what technology will look like in the next ten to twenty years. However, certain research directions could be essential for the evolution of the smart grid.

Increasing of the automation and decentralization of the grid will be a key field of research in the future. IoT technology can create smaller sections, or microgrids, that allow smart meters and substations to make changes in configuration based on user consumption levels, weather patterns and other predictive elements without the need for human interference or centralized decision making [43].

Another research aspect that should be focused on is reliability and integration testing. Since the grid is dependent on real-time data processing and the integration of various technologies, it is important to investigate the reliability of individual components and technologies, as well as the reliability of integrating these technologies in a homogenous system to provide recommendations for most reliable solutions to apply and identify fault areas to overcome. Similarly, since most of the technologies used in the smart grid were developed for other purposes, as well as the fact that the SG will have to adapt to future technology demands. It's worth investigating how new emerging technologies such as Nanotechnology or robotics can influence the development of SG systems.

\section{CONCLUSION}

Smart Grids are slowly replacing the traditional power grid to provide energy efficiency and reliability. As part of the fourth industrial revolution, SG is moving more and more in the direction of decentralization and automation. Thus, it is important to understand the technologies involved in the development and implementation of the SG as well as the challenges facing it.

The basic architecture of the SG includes three main layers (the power system layer, the communication layer, and the application layer) and uses different types of networks to facilitate communication between the different parts of the system (for example HANs, BANs, and WANs). In addition, SGs utilizes different components from various technologies such as IoT and Cloud computing in building the system.

Data collection and transmission are also important aspects of building a SG. There are new and existing technologies that could be utilized, like 5G Cellular networks and Fiber optics. Another important issue is integration of multiple devices and systems in one SG, which have seen few attempts to address it by introducing protocols such as DNP3 or OPC-UA. Moreover, Network management techniques such as SDN or virtualization can improve the performance of SG. The implementation of SG and expansion faces challenges due to its cyber-physical homogenous nature. These challenges include the lack of communication standards, signal interference, the need for real time data collection and processing, etc. All these issues and other security challenges like privacy and malicious attacks are problems that must be considered when working with SGs.

For the future, more research needs to be done on testing new and exciting technologies and techniques for automation, decentralization and integration. Future research can also explore new and promising technologies as possible solutions and answers to challenges facing the SGs.

\section{REFERENCES}

[1] D. Zhang, C. C. Chan, and G. Y. Zhou, "Enabling Industrial Internet of Things (IIoT) towards an emerging smart energy system," Global Energy Interconnection, vol. 1, pp. 39-47, 2018.

[2] V. C. Gungor, D. Sahin, T. Kocak, S. Ergut, C. Buccella, C. Cecati, et al., "Smart grid technologies: Communication technologies and standards," IEEE transactions on Industrial informatics, vol. 7, pp. 529-539, 2011.

[3] W. Wang, Y. Xu, and M. Khanna, "A survey on the communication architectures in smart grid," Computer networks, vol. 55, pp. 3604-3629, 2011.

[4] T. Stock and G. Seliger, "Opportunities of sustainable manufacturing in industry 4.0," Procedia Cirp, vol. 40, pp. 536-541, 2016.

[5] S. Chren, B. Rossi, and T. Pitner, "Smart grids deployments within EU projects: The role of smart meters," in 2016 Smart cities symposium Prague (SCSP), 2016, pp. 1-5.

[6] M. Moretti, S. N. Djomo, H. Azadi, K. May, K. De Vos, S. Van Passel, et al., "A systematic review of environmental and economic impacts of smart grids," Renewable and Sustainable Energy Reviews, vol. 68, pp. 888-898, 2017.

[7] R. Deng, Z. Yang, M.-Y. Chow, and J. Chen, "A survey on demand response in smart grids: Mathematical models and approaches," IEEE Transactions on Industrial Informatics, vol. 11, pp. 570-582, 2015. 
[8] M. Fischetti, G. Sartor, and A. Zanette, "MIP-and-refine matheuristic for smart grid energy management," International Transactions in Operational Research, vol. 22, pp. 49-59, 2015.

[9] V. C. Gungor, D. Sahin, T. Kocak, S. Ergut, C. Buccella, C. Cecati, et al., "A survey on smart grid potential applications and communication requirements," IEEE Transactions on industrial informatics, vol. 9, pp. 28-42, 2012.

[10] S. S. Reka and T. Dragicevic, "Future effectual role of energy delivery: A comprehensive review of Internet of Things and smart grid," Renewable and Sustainable Energy Reviews, vol. 91, pp. 90-108, 2018.

[11] N. S. Nafi, K. Ahmed, M. A. Gregory, and M. Datta, "A survey of smart grid architectures, applications, benefits and standardization," Journal of Network and Computer Applications, vol. 76, pp. 23-36, 2016.

[12] M. Ourahou, W. Ayrir, B. E. Hassouni, and A. Haddi, "Review on smart grid control and reliability in presence of renewable energies: Challenges and prospects," Mathematics and Computers in Simulation, 2018.

[13] Q.-D. Ho, Y. Gao, G. Rajalingham, and T. Le-Ngoc, "Wireless communications networks for the smart grid", vol. 2: Springer, 2014.

[14] M. Qasaimeh, R. Turab, and R. S. Al-Qassas, "Authentication techniques in smart grid: a systematic review," Telkomnika (Telecommunication, Computing, Electronics and Control), vol. 17, 2019.

[15] N. A. Abu Bakar, W. M. W. Ramli, and N. H. Hassan, "The internet of things in healthcare: an overview, challenges and model plan for security risks management process," Indonesian Journal of Electrical Engineering and Computer Science (IJEECS), vol. 15, pp. 414-420, July 20192019.

[16] Q. Ou, Y. Zhen, X. Li, Y. Zhang, and L. Zeng, "Application of internet of things in smart grid power transmission," in Mobile, Ubiquitous, and Intelligent Computing (MUSIC), 2012 Third FTRA International Conference on, 2012, pp. $96-100$

[17] D. Baimel, S. Tapuchi, and N. Baimel, "Smart grid communication technologies-overview, research challenges and opportunities," in Power Electronics, Electrical Drives, Automation and Motion (SPEEDAM), 2016 International Symposium on, 2016, pp. 116-120.

[18] N. Andreadou, M. Guardiola, and G. Fulli, "Telecommunication technologies for smart grid projects with focus on smart metering applications," Energies, vol. 9, p. 375, 2016.

[19] Y. Ma, F. Zhao, X. Zhou, and Z. Gao, "Summary of Cloud Computing Technology in Smart Grid," in 2018 IEEE International Conference on Mechatronics and Automation (ICMA), 2018, pp. 253-258.

[20] R. Ullah, N. Javaid, Z. Iqbal, I. Ahmad, A. Jan, and Y. K. Jadoon, "CRRP Analysis of Cloud Computing in Smart Grid," in Conference on Complex, Intelligent, and Software Intensive Systems, 2018, pp. 64-74.

[21] A. Morgado, K. M. S. Huq, S. Mumtaz, and J. Rodriguez, "A survey of 5G technologies: regulatory, standardization and industrial perspectives," Digital Communications and Networks, vol. 4, pp. 87-97, 2018.

[22] G. Shi and K. Li, "Fundamentals of ZigBee and WIFI," in Signal Interference in WiFi and ZigBee Networks, ed: Springer, 2017, pp. 9-27.

[23] S. S. Sabry, N. A. Qarabash, and H. S. Obaid, "The Road to the Internet of Things: a Survey," in 2019 9th Annual Information Technology, Electromechanical Engineering and Microelectronics Conference (IEMECON), 2019, pp. 290-296.

[24] D. G. Kogias, E. T. Michailidis, G. Tuna, and V. C. Gungor, "Realizing the Wireless Technology in Internet of Things (IoT)," in Emerging Wireless Communication and Network Technologies, ed: Springer, 2018, pp. 173-192.

[25] P. Sethi and S. R. Sarangi, "Internet of things: architectures, protocols, and applications," Journal of Electrical and Computer Engineering, vol. 2017, 2017.

[26] H. Yoo and T. Shon, "Challenges and research directions for heterogeneous cyber-physical system based on IEC 61850: Vulnerabilities, security requirements, and security architecture," Future generation computer systems, vol. 61, pp. 128-136, 2016.

[27] S. Cavalieri, M. G. Salafia, and M. S. Scroppo, "Integrating OPC UA with web technologies to enhance interoperability," Computer Standards \& Interfaces, vol. 61, pp. 45-64, 2019.

[28] S. Marksteiner, "Reasoning on Adopting OPC UA for an IoT-Enhanced Smart Energy System from a Security Perspective," in 2018 IEEE 20th Conference on Business Informatics (CBI), 2018, pp. 140-143.

[29] M. H. Rehmani, A. Davy, B. Jennings, and C. Assi, "Software Defined Networks based Smart Grid Communication: A Comprehensive Survey," arXiv preprint arXiv:1801.04613, 2018.

[30] M. Condoluci and T. Mahmoodi, "Softwarization and virtualization in 5G mobile networks: Benefits, trends and challenges," Computer Networks, vol. 146, pp. 65-84, 2018.

[31] M. Rihan, "Applications and Requirements of Smart Grid," in Smart Grids and Their Communication Systems, ed: Springer, 2019, pp. 47-79.

[32] G. Shi and K. Li, "Interference Avoidance in ZigBee Networks," in Signal Interference in WiFi and ZigBee Networks, ed: Springer, 2017, pp. 45-64.

[33] S. Singh and A. Yassine, "IoT Big Data Analytics with Fog Computing for Household Energy Management in Smart Grids," in International Conference on Smart Grid and Internet of Things, 2018, pp. 13-22.

[34] H. Daki, A. El Hannani, A. Aqqal, A. Haidine, and A. Dahbi, "Big Data management in smart grid: concepts, requirements and implementation," Journal of Big Data, vol. 4, p. 13, 2017.

[35] A. Malik and H. Om, "Cloud computing and internet of things integration: Architecture, applications, issues, and challenges," in Sustainable Cloud and Energy Services, ed: Springer, 2018, pp. 1-24.

[36] F. G. Bîrleanu, P. Anghelescu, N. Bizon, and E. Pricop, "Cyber Security Objectives and Requirements for Smart Grid," in Smart Grids and Their Communication Systems, ed: Springer, 2019, pp. 607-634. 
[37] H. Maziku, S. Shetty, and D. M. Nicol, "Security risk assessment for SDN-enabled smart grids," Computer Communications, vol. 133, pp. 1-11, 2019.

[38] R. Dorothy and S. Sasilatha, "Smart Grid Systems Based Survey on Cyber Security Issues," Bulletin of Electrical Engineering and Informatics (BEEI), vol. 6, pp. 337-342, 2017.

[39] A. Sanjab and W. Saad, "Data injection attacks on smart grids with multiple adversaries: A game-theoretic perspective," IEEE Transactions on Smart Grid, vol. 7, pp. 2038-2049, 2016.

[40] S. N. Narayanan, K. Khanna, B. K. Panigrahi, and A. Joshi, "Security in Smart Cyber-Physical Systems: A Case Study on Smart Grids and Smart Cars," in Smart Cities Cybersecurity and Privacy, ed: Elsevier, 2019, pp. 147-163.

[41] S. Armoogum and V. Bassoo, "Privacy of energy consumption data of a household in a smart grid," in Smart Power Distribution Systems, ed: Elsevier, 2019, pp. 163-177.

[42] A. Gusrialdi and Z. Qu, "Smart Grid Security: Attacks and Defenses," in Smart Grid Control, ed: Springer, 2019, pp. 199-223.

[43] E. O’Dwyer, I. Pan, S. Acha, and N. Shah, "Smart energy systems for sustainable smart cities: Current developments, trends and future directions," Applied Energy, vol. 237, pp. 581-597, 2019.

\section{BIOGRAPHIES OF AUTHORS}
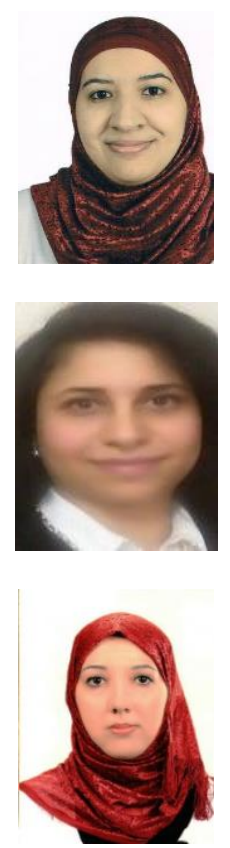

Noor Ahmed Qarabash is an assistant lecturer at the at the University of Information Technology and Communications in Baghdad, Iraq. She graduated with a Bachelor's degree in Information Technology Engineering in 2011 and obtained Master's degree in Cybersecurity and Management in 2014 from Warwick Univerisy. Her research interests include Cybersecurity, Data anyalsis, Internet of Things and Electronice governance. She can be contacted at noor.ahmed@uoitc.edu.iq

Sana Sabah Sabry was born in Basrah, Iraq in 1986. She received her B.Sc. degree in computer Engineering from Al-Nahrain University, Iraq in 2008 and her M.S. in Control Engineering from Al- Nahrain University, Iraq in 2011. She has 10 years' work experience at different educational organizations. She is currently a lecturer in the college of engineering at university of information technology and communications, Baghdad, Iraq. Her research topics include control theory, optimization algorithms and congestion control in computer networks, Internet of Things, Machine Learning. She can be contacted via email sana.sabah@uoitc.edu.iq.

Haneen Ahemd Qarabash is a lecturer at the Computing Science Department at the College of Science in the University of Baghdad. She graduated with a bachelor's in Computing Science in 2007 and obtained a master's degree in Web Development in 2009 from Al-Nahrain University. She was awarded with a Doctor of Philosophy (PhD) from Newcastle University in HumanComputer Interaction in 2018. Her research interests are on educational technology, students experience and engagement, alternative assessment, web development, human-computer interaction and user experience. She can be contacted at haneenqarabash@gmail.com 